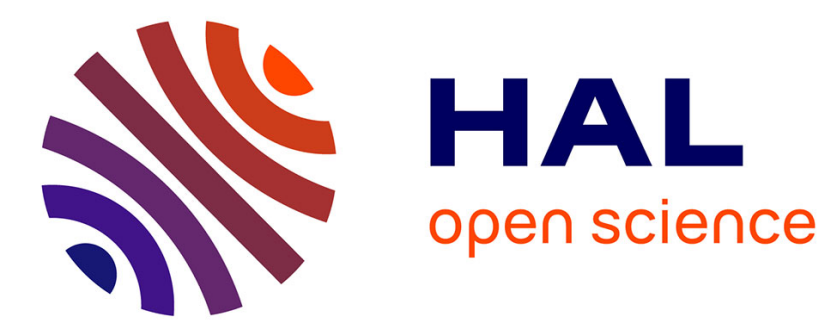

\title{
L'inscription arabe de Kilwa: nouvelle lecture
}

Saba Farès

\section{- To cite this version:}

Saba Farès. L'inscription arabe de Kilwa: nouvelle lecture. Semitica et Classica, 2010, 3, pp.241 248. 10.1484/J.SEC.1.100941 . halshs-01728353

\section{HAL Id: halshs-01728353 \\ https://shs.hal.science/halshs-01728353}

Submitted on 27 Sep 2021

HAL is a multi-disciplinary open access archive for the deposit and dissemination of scientific research documents, whether they are published or not. The documents may come from teaching and research institutions in France or abroad, or from public or private research centers.
L'archive ouverte pluridisciplinaire HAL, est destinée au dépôt et à la diffusion de documents scientifiques de niveau recherche, publiés ou non, émanant des établissements d'enseignement et de recherche français ou étrangers, des laboratoires publics ou privés. 


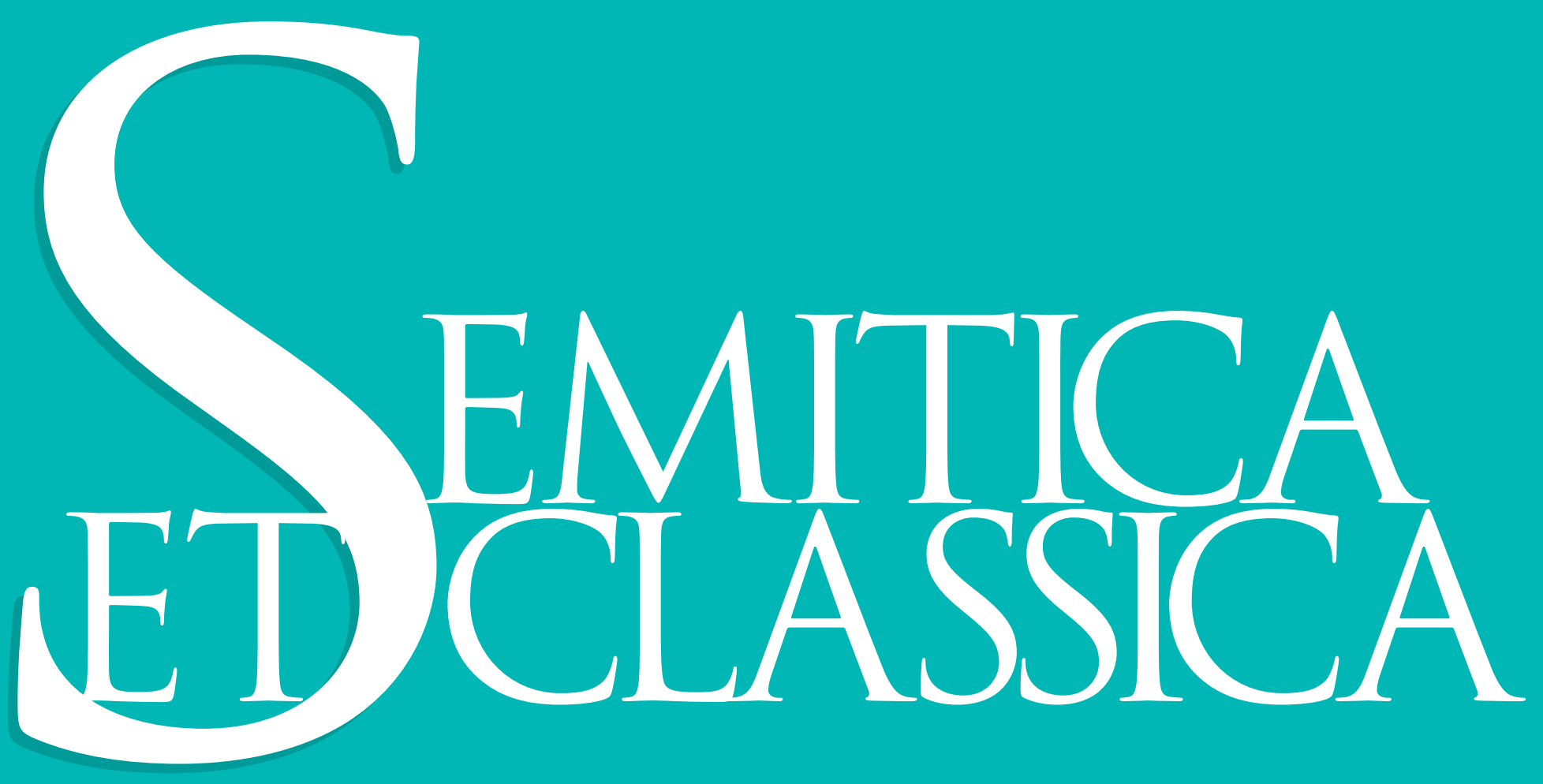

REVUE

INTERNATIONALE

D'ÉTUDES

ORIENTALES ET MÉDITERRANÉENNES

INTERNATIONAL JOURNAL OF ORIENTAL AND MEDITERRANEAN STUDIES
VOLVMEN III 2010

BREPOLS

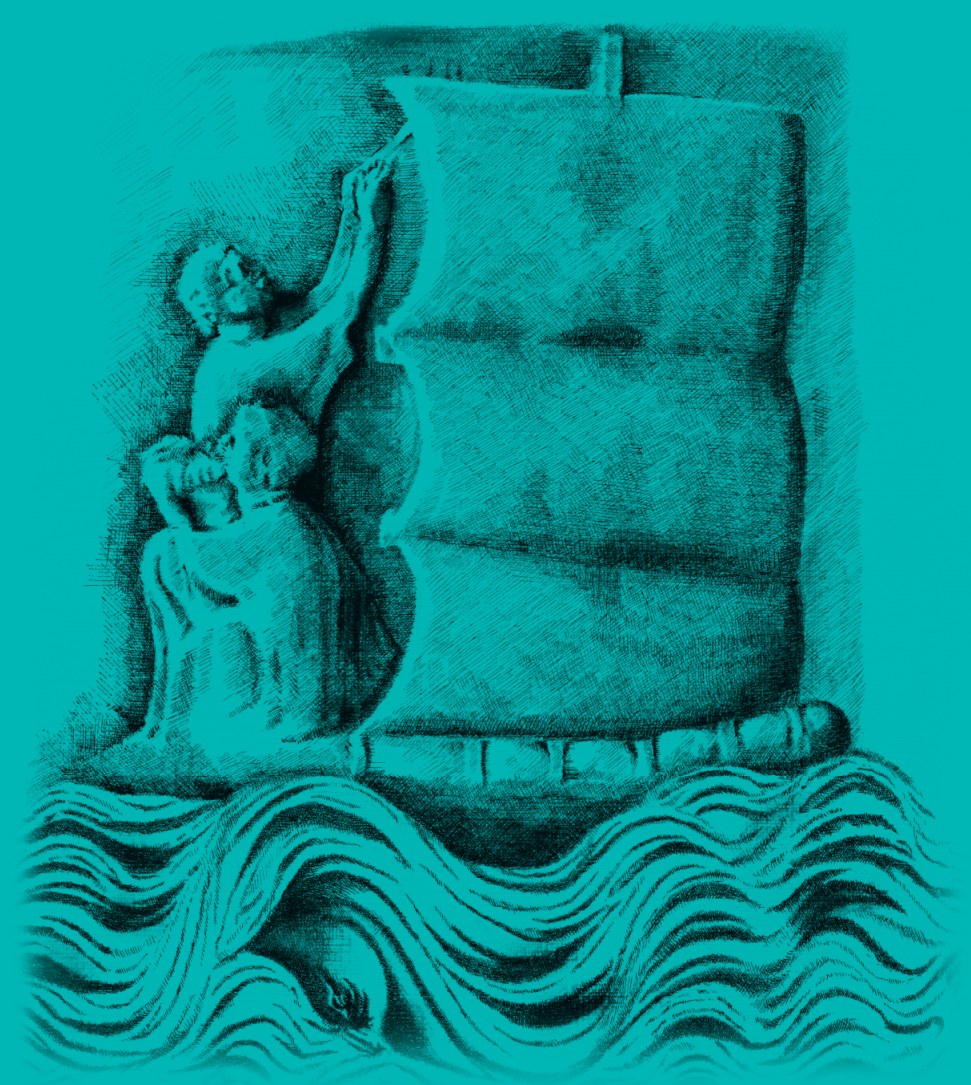




\section{SABA FARÈS L'inscription arabe de Kilwa : nouvelle lecture}

En novembre 1932, George Horsfield, alors directeur des Antiquités de Transjordanie, Agnes Horsfield et Nelson Glueck, alors directeur de l'American School of Oriental Research à Jérusalem, effectuent une exploration de cinq jours à Kilwa (Arabie). Les auteurs signalent la présence d'un monastère et de nombreuses croix ${ }^{1}$. Dans un premier article publié en 1933, ils donnent une très rapide description des vestiges et se concentrent sur les gravures rupestres préhistoriques ${ }^{2}$. Ils signalent néanmoins la présence, parmi les vestiges, d'une inscription en graphie arabe accompagnée d'une croix ${ }^{3}$. Cette inscription avait été signalée pour la première fois par G. Bell, passé par Kilwa, sur le chemin de Hâ'il ${ }^{4}$.

En 1936, N. Glueck publie une étude des vestiges archéologiques de Kilwa et les plans des bâtiments qui se trouvent au sud du site ${ }^{5}$. Il y donne une lecture de l'inscription, étudiée par L. A. Mayer de l'Université hébraïque. L. A. Mayer ne parvient à lire que la basmalah et avance la lecture de trois autres mots, mais tous sont erronés. Il propose de dater le texte de la fin $\mathrm{du} \mathrm{I}^{\mathrm{er}}$ millénaire après l'ère chrétienne.

Neuf ans plus tard, en 1943, A. Horsfield publie un article plus fourni où elle donne plus de détails sur cette inscription $^{6}$, après consultation du Père dominicain Savignac, pour qui la graphie serait aussi de la fin du ${ }^{\text {er }}$ millénaire ${ }^{7}$.

Depuis 2008, nous avons repris le travail de nos prédécesseurs : nous avons entamé des fouilles dans le monastère (figure 1) et des prospections archéologiques et épigraphiques dans une zone de $40 \mathrm{~km}$ de diamètre ${ }^{8}$.

1. Horsfield, Horsfield, Glueck 1933, p. 381-386.

2. Ibidem.

3. Ibid., p. 381.

4. Rapportée d'abord par HoRSFIELD 1943, p. 73-74, d'après les informations données par la sœur de G. Bell, publiées par O'BRIEN 2000.

5. GLUCK 1935, p. 9-16.

6. HORSFIELD 1943, p. 74

7. Ibidem, p. 74.

8. Un projet franco-saoudien a été lancé en 2009 sous le patronage de la Sous-Direction des Sciences humaines
Bien entendu, nous avons cherché la fameuse inscription arabe et nous l'avons retrouvée (figures 2 et 3). Elle est restée intacte, en très bon état, pas du tout détériorée depuis le passage de l'équipe anglaise.

L'inscription est gravée sur le linteau de la porte d'une cellule, qui porte le $\mathrm{n}^{\circ} 63$ dans notre inventaire. Le linteau mesure $1,20 \mathrm{~m}$ de long sur $0,30 \mathrm{~m}$ de hauteur. Dans la partie droite a été dessinée une croix aux bras en forme de triangle. À gauche se trouve l'inscription. Elle est gravée sur une surface préparée, des traces d'outil sont visibles, formant une sorte de cartouche. Le texte a résisté à la lecture pendant très longtemps, probablement parce que les différentes tentatives n'avaient pour support iconographique qu'une photographie, ce qui rend la lecture difficile faute d'avoir vu le texte in situ, à cause des traces d'outils sur la pierre qui peuvent prêter à confusion. Après notre première visite à Kilwa et après y avoir passé plusieurs jours, j'ai réussi à lire le texte, mais le problème était sa compréhension. À ce jour, trois expéditions ont eu lieu et beaucoup de réflexions ont été menées sur le fonctionnement du site lui-même, ce qui me conduit aujourd'hui à proposer la lecture suivante :

$$
\text { بسم الله حمة أهل تكلا من إقليم... }
$$

Bism Allâh himat 'hl Taklâ min 'Iqlîm

Au nom de Dieu, (ceci est) le territoire protégé de la communauté de Thècle, originaire d'Iqlîm

L. A. Mayer avait proposé à N. Glueck la lecture suivante :

$$
\begin{aligned}
& \text { وبسم الله عمل هذ... قلي } \\
& \text { in the name of God. Made this... cell }{ }^{9}
\end{aligned}
$$

et de l'archéologie du ministère des Affaires étrangères français, sous la direction de $\mathrm{S}$. Farès. Deux campagnes ont eu lieu à ce jour. La campagne 2010 a révélé l'existence d'une église dont la publication est en cours.

9. GLUECK 1935, p. 14-15. 


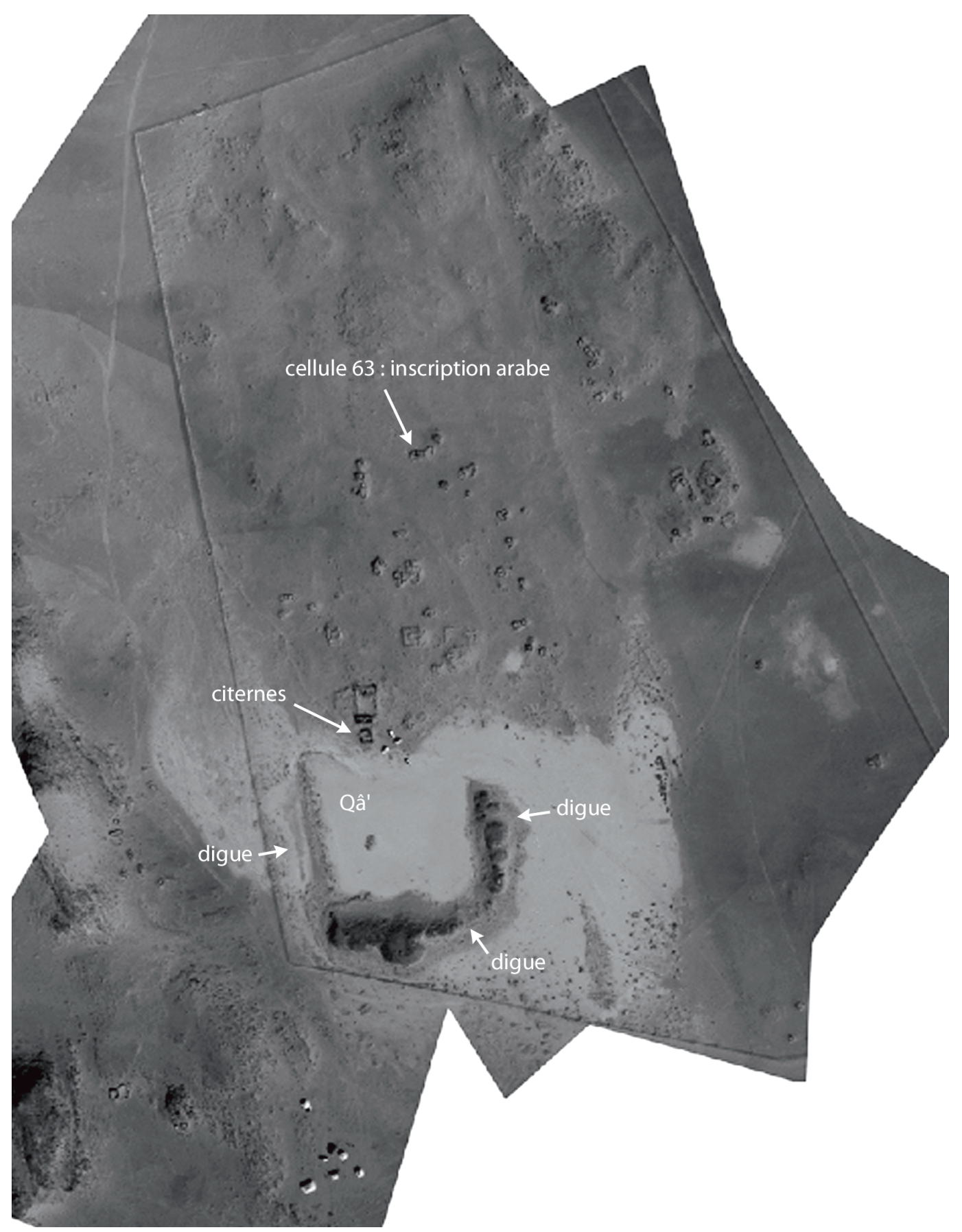

Figure 1 - Vue générale du monastère ;

emplacement de l'inscription et des aménagements hydrauliques. 


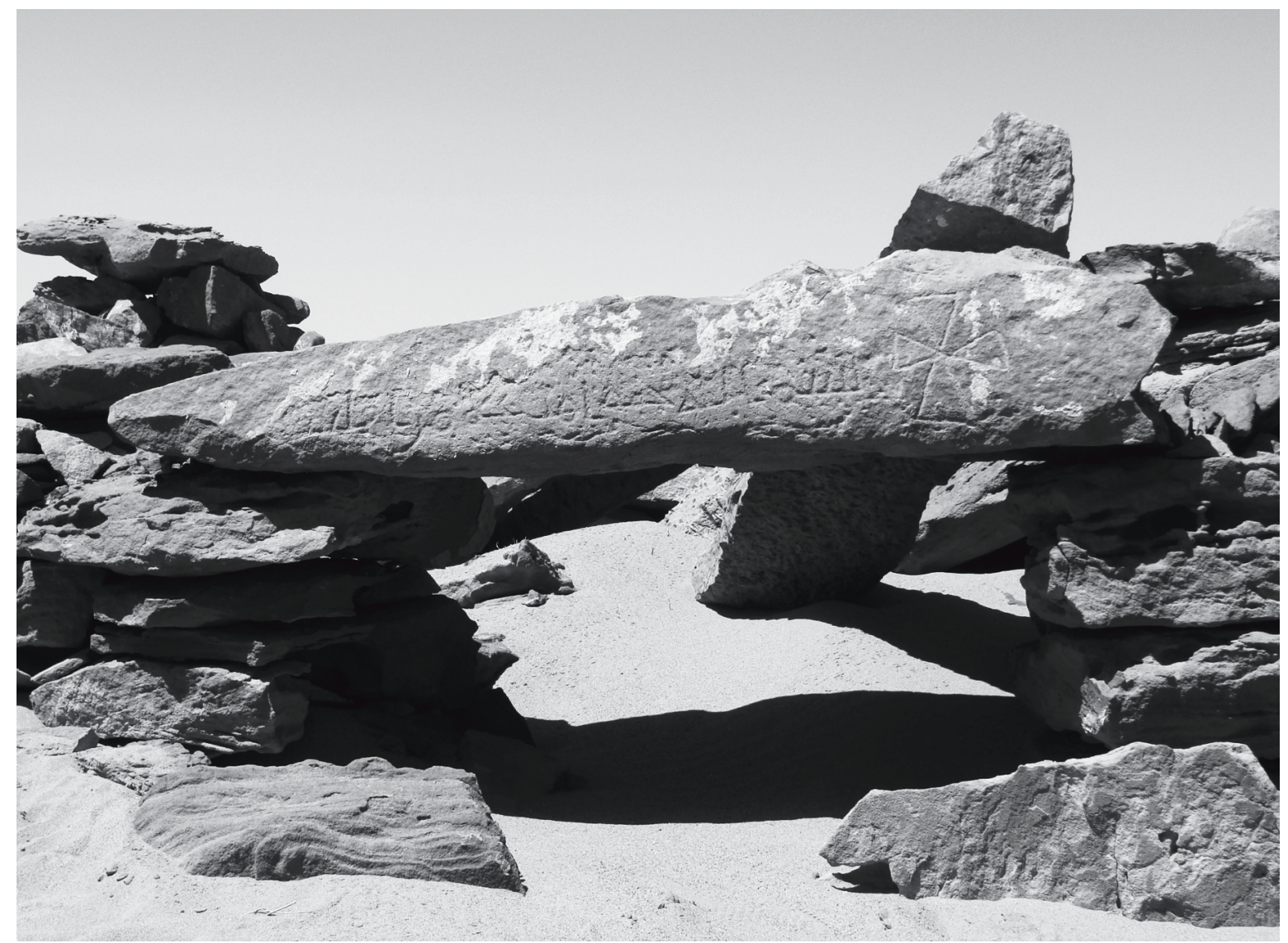

Figure 2 - L'inscription de Kilwa.

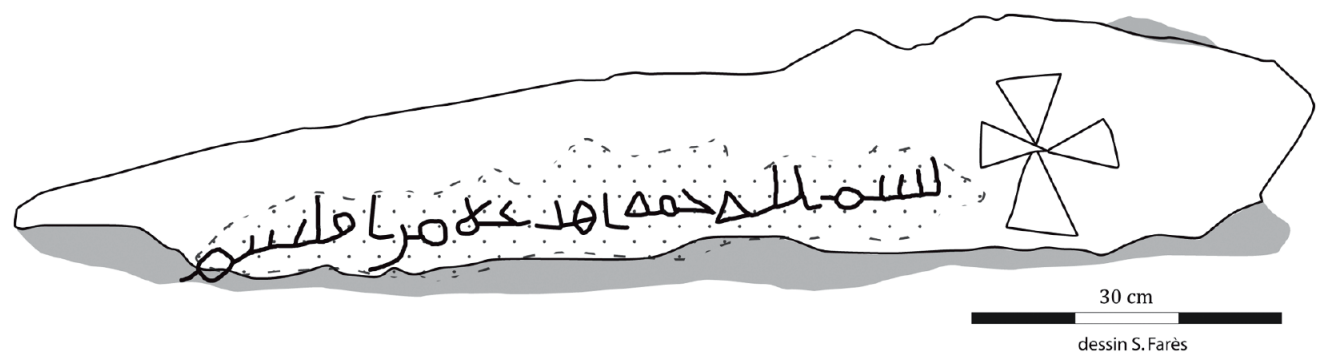

Figure 3 - Fac-similé de l'inscription. 
Le texte ne présente pas de difficulté majeure de déchiffrement des lettres qui sont en bon état général, sauf celles du dernier mot, la dernière lettre étant légèrement effacée. Les traces d'outils sur la pierre et l'érosion peuvent prêter à confusion sur la photographie. Contrairement à ce que ces traces peuvent indiquer, l'inscription ne porte aucun signe diacritique.

La basmalah peut induire un doute sur la confession de l'auteur du texte : compte tenu du contexte chrétien toutefois, elle est sans équivoque. Elle ne suit pas la formule habituelle complète : au nom de dieu le clément le miséricordieux. Celle-ci est connue par un graffiti chrétien daté de 481 de l'hégire / 1058 de l'ère chrétienne, qui se trouve dans le couvent de Mar Moussa, près du Nabk, en Syrie. Selon un prêtre de ce couvent, elle apparaît également dans des manuscrits syriaques de l'époque antérieure à $l^{\prime}$ 'islam ${ }^{10}$. Une autre inscription chrétienne commence également par la basmalah : à Șadad, à $60 \mathrm{~km}$ à l'est de Homs, en Syrie, datée de 1153 de l'ère chrétienne (figure 4) ${ }^{11}$.

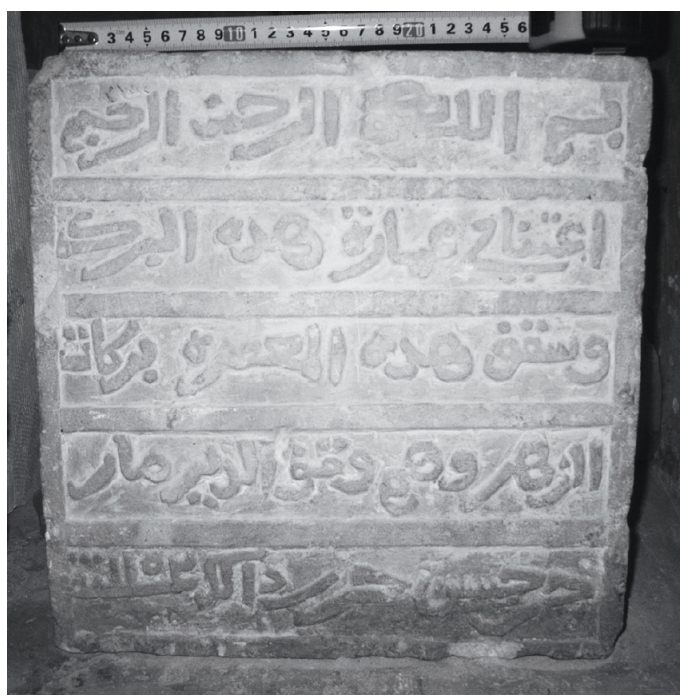

Figure 4 - Inscription arabe de Șadad (S. Farès).

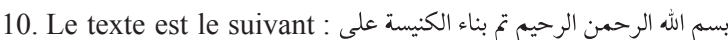
au nom de dieu le clément le miséricordieux, la construction de cette église a été achevée à l'époque de Thomas le nabkî, fils d'al-Assad. Source: http://www.nobles-news.com/news/news/index.php? page $=$ show det $\&$ select page $=12$ \&id $=67046$.

11. Cette inscription provient de l'église Saint Georges, du rite syriaque-orthodoxe, que j'ai visitée en juillet 2010. Elle

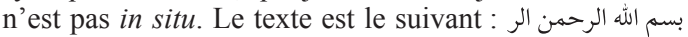

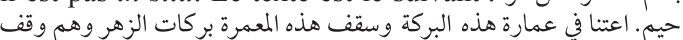

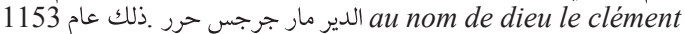
le miséricordieux, Barakat al-Zahr s'est occupé de la construction de ce puits et de la couverture de ce bâtiment qui sont la propriété du couvent Saint George. Le texte a été rédigé en l'an 1153.
- himat signifie en arabe « le territoire interdit, une réserve »; il est employé, selon Lisân al- 'arab, pour désigner une zone protégée destinée à une exploitation agricole, au pâturage des chameaux, ou bien une zone qui recueille la pluie et réservée à un groupe de personnes qui gèrent l'exploitation ${ }^{12}$.

- 'hl: en arabe le mot a le sens de «population, communauté »).

- Taklâ : la lecture de ce nom est claire, elle ne présente aucune difficulté ; Taklâ rappelle le nom de « Thècle », la sainte disciple de l'apôtre Paul, qui apparaît pour la première fois dans les Actes de Paul et de Thècle, à la seconde moitié du $\mathrm{II}^{\mathrm{e}}$ siècle $^{13}$, et dont le culte a connu une grande diffusion en Séleucie et en Syrie, $\mathrm{au} \mathrm{IV}^{\mathrm{e}}$ et au $\mathrm{V}^{\mathrm{e}}$ siècles de notre ère ${ }^{14}$. Le monastère le plus connu, dédié à cette sainte, se trouve à Ma'aloula, en Syrie, à $50 \mathrm{~km}$ au nord de Damas.

- min: préposition sémitique indiquant l'origine; d'après $\mathrm{C}$. Brockelmann, la large utilisation de cette préposition dans ce sens en a fait la préposition de l'《 identité $»^{15}:$ min indique la parenté, l'origine, la place d'une personne dans un groupe (tribu, famille), dans un lieu ${ }^{16}$

- 'Iqlîm: L. A. Mayer n'avait pas vu le alif et avait lu un tâ marbûta à la place du mîm. Il a bien identifié le $q \hat{a} f$ et le $y \hat{a}$. La dernière lettre est légèrement érodée, mais toutes les autres lettres sont bien lisibles. On s'attendrait à trouver ici le nom d'origine de la communauté. Le mot signifie en arabe "province, district, territoire ». 'Iqlîm chez Yaqût al-Hamwî est le nom d'une région près de Damas ${ }^{17}$. Ce toponyme est mentionné par al-Qalânisî, dans Dayl ta'rîh Dimašq et sa description de la campagne du Sultan Nûr ad-Dîn Zankî contre les Croisés et de son entrée à Damas. Selon al-Qlânisî, al-'Iqlîm (الإقليم) serait le lieu où se sont réfugiés les infidèles lorsqu'ils ont vu l'importance de l'armée du Sultan ${ }^{18}$. Selon H. A. R. Gibb, le lieu qui pourrait le mieux correspondre à la description d'al-Qalânisî serait ce qu'on appelle aujourd'hui 'Iqlîm Ballân, nom qui désigne une région qui se trouve le long du Nahr al-A 'waj ${ }^{19}$.

12. Le système de Hima est connu dans les zones arides et fait l'objet d'une littérature assez abondante. Pour plus de détails sur ce système, voir BocCo 1993, p. 349-353, et Draz 1985, p. 109-121.

13. Festugière 1968, p. 52.

14. DAVIS 2001, p. 4.

15. BROCKELMANN 1908, II, p. 397.

16. BLACHÈRE 1975 , p. 339.

17. AL-HAMWî 1993, t. 1, p. 237

18. Al-QLÂNISî 1983, p. 351

19. Cf. GiBB 2002 , p. 345 , n. 1 . Une rivière qui prend sa source dans le Jabal aš-Šayh, passe par Damas et termine sa course dans le Sabhat al-Hayjâna. 


\section{REMARQUES GÉNÉRALES}

La première observation porte sur la graphie. Au premier coup d'œil, elle évoque celle qui a connu une grande diffusion au Bilâd ash-Shâm à partir de la seconde moitié du VII ${ }^{\mathrm{e}}$ siècle $^{20}$. Le contexte et l'environnement du site évoquent Qasr Burqu', en Jordanie ${ }^{21}$. La graphie de notre texte est d'un style identique à celle de Burqu', datée de l'an 81 de l'hégire / 700 de l'ère chrétienne ${ }^{22}$. Il s'agit d'un texte de construction commandité par le calife al-Walid, cinq ans avant son accession au pouvoir (705-715). Dans ce texte, la basmalah est complète, selon la formule musulmane : bismillah ar-rahmân ar-rahîm. La graphie de notre inscription serait alors, par comparaison, antérieure au VIII ${ }^{\mathrm{e}}$ siècle. Les céramiques trouvées à Kilwa, dans la fouille et en contexte, appuient cette datation ${ }^{23}$.

L'utilisation de la graphie arabe par une communauté chrétienne ne semble pas étonnante, ni même l'emploi de la basmalah. La communauté chrétienne melkite avait adopté la graphie arabe dans la liturgie ${ }^{24}$ et cette graphie a fini par être le symbole identitaire de cette communauté $^{25}$. Avant même que cette écriture ne devienne celle des Melkites et de la religion de l'islam, elle a été celle des tribus arabes de la Syrie, puisque la graphie de ce texte évoque certains aspects de l'inscription de Jabal Sâys ${ }^{26}$, tels que le alif et le alif-lâm, le mîm, etc. Il faut rappeler que les inscriptions les plus anciennes en graphie arabe, connues à ce jour, sont au nombre de six : an-Namâra (328 de l'ère chr.), Zabad (au sud d'Alep, 512 de l'ère chr.), Harrân (538 de l'ère chr.), Jabal Says (528 de l'ère chr.), Umm al-Jimâl (Jordanie du Nord, datation incertaine), et Jabal Ramm (Jordanie du Sud, datation incertaine). Quatre ont été trouvées dans le Hûrân (désert basaltique syro-jordanien) : an-Namâra, Harrân, Jabal Sâys et Umm al-Jimâl, dont

20. ORY 2009, p. 344

21. Qasr Burqu' est une bourgade sur la route de MafraqBaghdad, au nord-est de la Jordanie. Il s'agit des vestiges qui indiquent, selon Gaube, quatre périodes d'occupations (construction ou re-construction) (GAUBE 1974, p. 98). Pour la bibliographie complète, voir GAUBE 1974.

22. Cette inscription a été incomplètement publiée dans le Répertoire chronologique d'épigraphie arabe. Elle a été complétée par H. Field avec l'aide de H. A. R. Gibb (FIELD 1960, p. 15, dessin p. 155). Elle a été reproduite d'après la lecture de H. Field, par GAUBE 1974, p. 97.

23. Les céramiques trouvées dans la fouille sont étudiées par Marie-Odile Rousset. Le rapport de l'étude est en cours de publication.

24. GRIFFITH 1989, p. 7.

25. GRIFFITH 2001, p. 16 ; IDEM 2002, p. 24.

26. Inscription datée de 528-529 de l'ère chrétienne. Pour une bibliographie complète de l'inscription et la dernière analyse du texte, voir RoBIN, GOREA 2002, p. 503-510. trois dans des églises : Harrân, Umm al-Jimâl, Zabad. Une seule sort du lot, celle de Jabal Ramm (Jordanie du Sud, IV siècle de l'ère chr. $)^{27}$.

La deuxième observation porte sur le contenu de l'inscription. Le texte mentionne la présence d'une himat, une réserve destinée à recueillir l'eau de pluie et à l'agriculture. Ceci correspond parfaitement aux éléments architecturaux du site et aux données archéologiques mises en évidence par notre projet.

Le site de Kilwa est construit aux bords d'un $q \hat{a}$ ', un lac d'eau douce artificiel. Le monastère s'appuie donc surtout sur un système hydraulique très élaboré constitué d'une digue retenant l'eau de pluie qui descend des wadis et se verse dans une cuvette naturelle formant un lac une fois remplie. Il faut y ajouter une citerne, composée d'un double bassin qui récupère l'eau de pluie directement par les toitures ou par des canaux aménagés recueillant l'écoulement des wadis (figure 5). La fouille, en 2010, d'un espace carré muré mesurant $15 \mathrm{~m}$ de côté et rempli de cendres a livré une grande quantité de noyaux de fruits, tels que des prunes et des pêches, des olives et des dattes, ce qui suppose que des arbres fruitiers, des oliviers et des dattiers ont été cultivés sur place. L'importance de l'agriculture dans la vie monastique en Syrie est bien connue ${ }^{28}$; si protéger le territoire paraît normal, surtout dans une région très aride, où l'eau est précieuse, cultiver des fruits dans un tel environnement relève de l'exploit.

Enfin, l'hypothèse selon laquelle le site aurait été un poste romain-byzantin est exclue ${ }^{29}$. Il est certain aujourd'hui qu'il s'agit d'un établissement chrétien : la quantité de croix, le dégagement d'une église par notre équipe en 2010 et la chapelle renforcent cette hypothèse. Il faut y ajouter l'organisation des bâtiments et leur fonctionnement qui sont ceux d'un monastère : cellules isolées pour des moines, chapelle, église, cuisine,

27. À propos de ces inscriptions, voir le travail le plus récent sur la question : WerNER Diem 1976, p. 254 ; Chr. Robin a fait un état de la question en 2001 (RoBIN 2001, p. 509577). Concernant les inscriptions de Jabal Usays et de Harrân, cf. SHAHID 1995, respectivement p. 117-124 et 325-331; à propos de celles de Jabal Ramm et Umm al-Jimâl, voir Bellamy 1988, p. 369-378. L'inscription d'an-Namâra a été publiée pour la première fois par Dussaud 1902, II, p. 409-421; elle a connu ensuite de nombreuses publications : on doit le dernier commentaire à RoBIN 1991, p. 11 et 116-117 ; elle se trouve dans le Répertoire d'épigraphie sémitique, $\mathrm{n}^{\circ} 483$. L'inscription de Zabad est publiée dans le Répertoire chronologique d'épigraphie arabe, où elle porte le $\mathrm{n}^{\circ} 2$, p. 2-3. Enfin, l'inscription de Harrân porte le $\mathrm{n}^{\circ} 3$ dans le même Répertoire chronologique d'épigraphie arabe, p. 3-4.

28. Voir à titre d'exemple Deir Turmanin, en Syrie du Nord : BRENK 2004, p. 463-464.

29. SARTRE 1981, p. 86. 


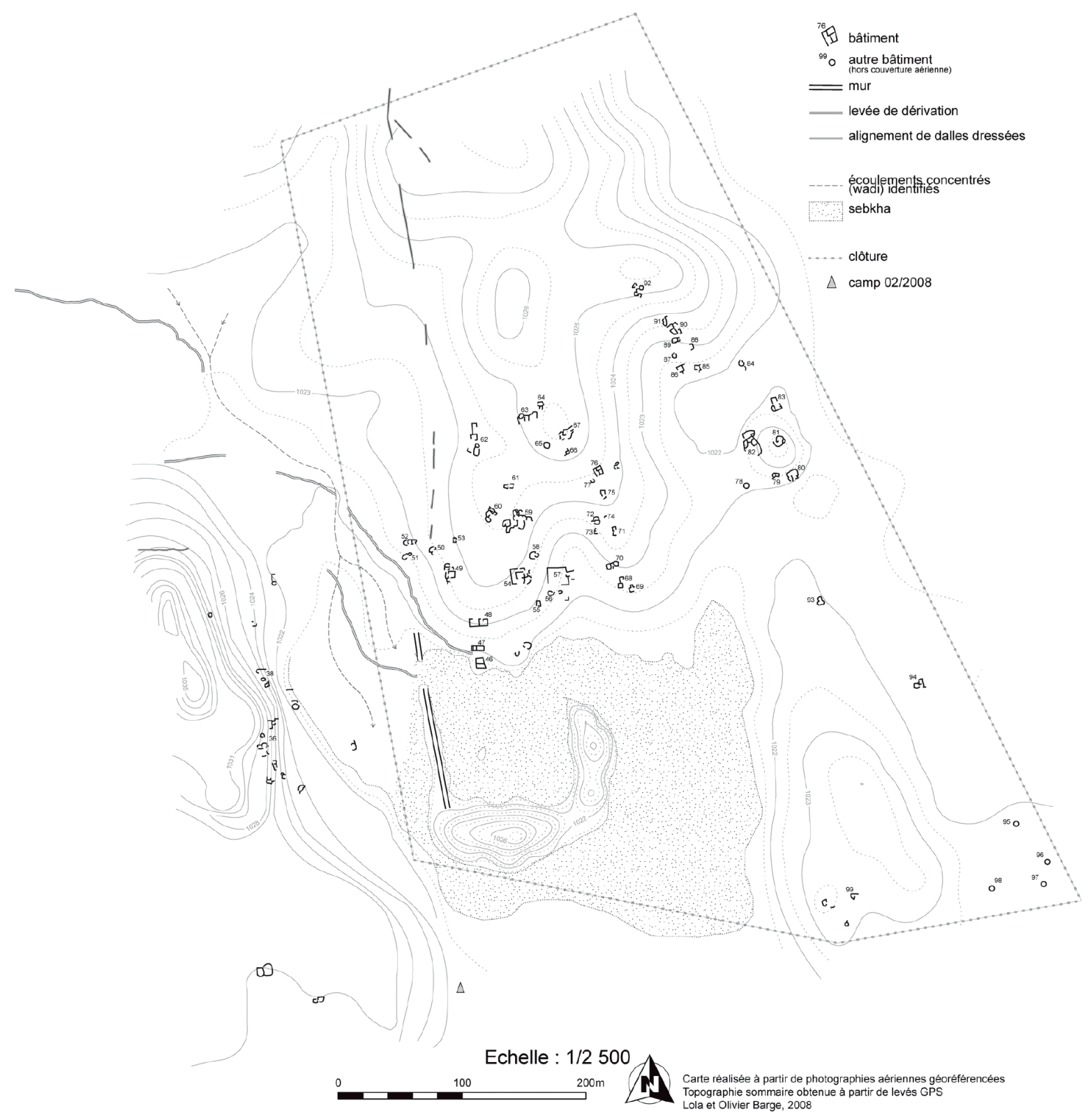

Figure 5 - Relevé topographique du monastère et système de canalisation d'eau de pluie. 
réfectoire, citernes d'eau et jardins. Le nom même du site est intéressant à observer : Kilwa en arabe signifie « isolement, solitude, réclusion, l'endroit où on est en

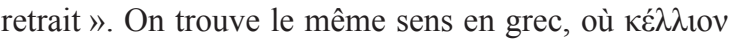
désigne « le lieu de méditation $»^{30}$.

En conclusion, on pourrait souligner que plusieurs éléments nous orientent vers une communauté monastique d'origine syrienne. La sainte patronne du lieu, sainte Thècle, dont le culte est répandu en Syrie, le toponyme et l'architecture sont des détails qui indiquent une culture syrienne. La graphie vient s'ajouter à ces éléments et renforce l'hypothèse de l'origine syrienne de cette communauté. Ce monastère est le plus au sud du monde chrétien byzantin connu à ce jour et le plus à l'ouest du monde chrétien perse. Les fouilles à venir pourraient apporter des réponses aux questions posées par cette inscription.

Qu'une communauté chrétienne soit entourée d'une communauté musulmane est une pratique assez courante. Ibn Jubair, au XII ${ }^{\mathrm{e}}$ siècle, dans l'ouvrage qui relate son voyage à la Mecque, mentionne la présence, en Syrie, de nombreux monastères et villages appartenant à des chrétiens entourés d'Arabes musulmans avec qui ils ont conclu des accords ${ }^{31}$. La communauté installée à Kilwa ne devait pas être étrangère aux habitants de la région pour avoir pu s'y installer. La gestion des ressources dans le désert est régie par des réglementations assez strictes. Accéder aux ressources naturelles nécessite des accords entre les populations. À Dîsseh, près de Ramm, en Jordanie du Sud, une inscription en langue arabe et écriture nordarabique, précise les noms des personnes qui contrôlent la distribution de l'eau récoltée par ruissellement dans une grotte ${ }^{32}$. Cette pratique est encore aujourd'hui en vigueur dans le désert, à Ramm notamment ${ }^{33}$.

Au total, si la communauté installée à Kilwa semble être de culture syrienne, elle n'est pas étrangère au monde nomade. Ses membres ont pu tirer profit des techniques venant de la Syrie pour exploiter un territoire

30. HoRSFIELD 1943, p. 74

31. Le voyage d'Ibn Jubair a été révisé et traduit en anglais par WRIGHT 1907, puis par BROADHURST 1952. Ibn Jubair (1145-1217) était un géographe, voyageur et poète andalous qui effectua trois voyages en Orient : son ouvrage ne relate que son premier voyage.

32. Cf. FARĖs-DraPpeAu 1995, p. 493-497.

33. Observé durant nos campagnes de prospections archéologiques et épigraphiques à Ramm, en Jordanie du Sud, dans le cadre du projet Franco-Jordanie. Ce projet, commencé en 1995, continue encore aujourd'hui grâce au financement de la Sous-direction des Sciences humaines et de l'archéologie du ministère des Affaires étrangère français, et est dirigé par S. Farès, F. Zayadine et Kh. Al-Jbour (Département des Antiquités de Jordanie). très aride. Les recherches à venir pourraient nous éclairer davantage sur cette communauté et les techniques employées pour survivre dans un tel milieu.

Université Nancy 2

\section{BIBLIOGRAPHIE}

BELL G.

1914 «A Journey in Northern Arabia », The Geographical Journal 44/1, p. 76-77.

Bellamy J. A.

1988 «Two Pre-Islamic Arabic Inscriptions Revised: Jabal Ramm and Umm al- Jimal », Journal of the American Oriental Society 108, p. 369-378.

Blachère R., Gaudefroy-Demombynes M.

1975 Grammaire de l'arabe classique, Paris, Maisonneuve Larose éd.

Bocco R.

1993 « De la sécurité politique à l'autonomie alimentaire dans les steppes : experts internationaux et programmes de développement (1950-1990)», dans R. Bocco, R. Jaubert, F. Métral (éd.), Steppes d'Arabie, États, pasteurs, agriculteurs et commerçants : le devenir des zones sèches, Genève (Cahier de l'IUED), p. 326-353.

BRENK B.

2004 «Monasteries as rural settlements », dans W. A. BowDen, L. Lavan, C. Machado (éd.), Recent Research on the Late Antique Countryside, Leyde, Brill.

BROADHURST R. J.C.

1952 IBn JUbaYR, The Travels of Ibn Jubayr, trad. et éd. R. J. C. Broadhurst, Londres, Cape.

BROCKELMANN C.

1908 Grundriss der vergleichenden Grammatik der semitischen Sprachen (2 volumes), Berlin.

DAVIS S.J.

2001 The Cult of Saint Thecla: A Tradition of Women's Piety in Late Antiquity, Oxford, Oxford University Press.

DIEM W.

1976 « Some Glimpses at the Rise and Early Development of the Arabic Orthography », Orientalia 45, p. 251-261.

DRAZ O.

1985 « The Hema System of Range Reserves in the Arabian Peninsula. Its Possibilities in Range Improvement and Conservation Projects in the Near East », dans J. A. MCNeely, D. C. PitT (éd.), Culture and Conservation: The Human Dimension in Environmental Planning, Worceste, Billing and Sons Limited, p. 109-121.

Dussaud R.

1902 «Inscription Nabatéo-Arabe d'en-Namâra », Revue archéologique II, p. 409-421. 
FARÈs-DRAPPEAU S.

1995 «L'inscription de type dédanite de Abu Ad-Diba“ Wadi Ramm, une nouvelle lecture », ADAJ 39, p. 493-497.

Festugière A.-J.

1968 «Les énigmes de sainte Thècle », Comptes-rendus de l'Académie des Inscriptions et des Belles-Lettres, $112^{\mathrm{e}}$ année, N. 1, p. 52-63.

FIELD H.

1960 North Arabian Desert Archaeological Survey 192550, Cambridge, Massachusettes (Peacbody Museums Papers XLV, 2).

GAube H.

1974 «An Examination of the Ruins of Qasr Burqu“ », Annual of the Department of Antiquities of Jordan 19, p. 93-100.

GiBB H. A. R.

2002 The Damascus Chronicle of the Crusades: Extracted and Translated from the Crusades, Extr. and transl. from the Chronicle of Ibn al-Qalânisî, New-York, Dover edition.

GLUCK N.

1935 «Christian Kilwa», Journal of the Palestine Oriental Society 16, p. 9-16.

GRIFFITH S.H.

1989 « Anthony David of Baghdad, Scribe and Monk of Mar Sabas: Arabic in the Monasteries of Palestine », Church History 58/1, P. 7-19.

2001 "'Melkites', 'Jacobites' and the Christological Controversies in Arabic in Third/Ninth Century », dans D. Thomas (éd.), Syrian Christians under Islam: The First Thousand Years, Leyde, Brill, p. 9-55.

2002 "From Aramaic to Arabic: The Language of the Monasteries of Palestine in the Early Islamic Period » (Dumbarton Oaks Papers, 51 [1997]), dans The Beginnings of Christian Theology in Arabic: Muslim-Christian Encounters in the Early Islamic period (Collected Studies Series 746), Aldershot, Hamwî (al-) Y .

1993 Mu jam al-Buldân (5 tomes), Beyrouth, éd. Dâr Șâdir.

HORSFIELD A.

1943 « Journey to Kilwa, Transjordan », The Geographical Journal 102/2, p. 71-77.

Horsfield G., Horsfield A., Glueck N.

1933 «Prehistoric Rock-Drawings in Transjordan", American Journal of Archaeology 37/3, p. 381-386.
IBN AL-QALÂNISî, ABU YA'LA HAMZAH IBN AsAD

1983 Dayl ta'rh Dimašq, étudié par Souhayl Zakkâr, éd. Dâr Hassân.

IBN MANZ̛UR, MuHAMMAd IBN MAKRAM 1993 Lisân al- 'arab, 15 vols., Beyrouth, Dâr Șâdir.

O'BRIEN R.

2000 Gertrude Bell: The Arabian Diaries, 1913-1914, USA, Syracuse University Press.

ORY S.

2009 « La graphie des inscriptions arabes avant l'Islam et à l'époque umayyade », dans K. BARTL, A. MOAZ (éd.), Residences, Castles, Settlements (OrientArchäologie, Band 24), p. 339-356.

Répertoire Chronologique d'Épigraphie Arabe, dir. Ét. COMBE, J. Sauvaget et G. Wiet, Le Caire, IFAO.

Répertoire d'Épigraphie Sémitique, dir. J.-B. Chabot, Académie des Inscription et des Belles-Lettres, Commission du Corpus Inscriptionum Semiticarum [t. V, G. RYCKMANS, (n 2624-3052), 1929 ; t. VI, IDEM (nº 3053-3946), 1935 ; t. VII, IDEM, (n $3947-5106)$, 1936-1950 ; t. VII, J. PirenNe, Tables et index des RoBIN Chr. t. V, VI, VII, 1968], Paris, Imprimerie nationale.

1991 «L'Arabie antique de Karib'il à Mahomet (nouvelles données sur l'histoire des Arabes grâce aux inscriptions) », Revue du Monde musulman et de la Méditerranée 61/3.

2001 « Les inscriptions de l'Arabie antique et les études arabes », Arabica 48/4, p. 509-577.

RoBin Chr., Gorea M.

2002 «Un réexamen de l'inscription arabe préislamique du Ğabal Usays (528-529 è. chr.) », Arabica 49/4), p. $503-510$

SARTRE M.

1981 «La frontière méridionale de l'Arabie romaine », dans La géographie administrative et politique d'Alexandre à Mahomet (Actes du colloque de Strasbourg, 14-15 juin 1979), p. 77-92.

SHAHID I.

1995 Byzantium and the Arabs in the Sixth Century, vol. I, 1. Political and Military History, Washington, Dumbarton Oaks Research Library and Collection. WRIGHT W.

1907 Ibn Jubair, The Travels of Ibn Jubayr, trad. et éd. W. Wright, rev. M. J. de GoEJE (révision de l'édition de 1852), Leyde, Brill. 